\title{
Oxidative Stress Response in Patients Infected by Diverse Hepatitis C Virus Genotypes
}

\author{
Mohammad Hassan Khadem Ansari ${ }^{1}$; Mir-Davood Omrani ${ }^{2}$; Fatemeh Kheradmand ${ }^{3, *}$ \\ ${ }^{1}$ Clinical Biochemistry Department, Faculty of Medicine, Urmia University of Medical Sciences, Urmia, IR Iran \\ ${ }^{2}$ Medical Genetics Department, Faculty of Medicine, Shahid Beheshti University of Medical Sciences, Tehran, IR Iran \\ ${ }^{3}$ Cellular and Molecular Research Center, Clinical Biochemistry Department, Faculty of Medicine, Urmia University of Medical Sciences, Urmia, IR Iran \\ ${ }^{*}$ Corresponding Author: Fatemeh Kheradmand, Cellular and Molecular Research Center, Clinical Biochemistry Department, Faculty of Medicine, Urmia University of Medical Sci- \\ ences, 11th Km of Sero (Nazloo) Road, Urmia, IR Iran. Tel:+98-4412770397, Fax:+98-4412780800, E-mail: fkheradmand@umsu.ac.ir
}

Received: July 15, 2014; Revised: October 12, 2014; Accepted: February 1, 2015

\begin{abstract}
Background: The molecular mechanism of hepatitis C-virus (HCV) genome-specific pathogenesis remains unclear. Oxidative stress is an important pathophysiological mechanism in chronic HCV infection, but its relation to HCV genotypes has not been thoroughly examined. Objectives: In the present case-control study, the effect of diverse HCV genotypes on oxidative status changes was investigated.

Patients and Methods: From 310 patients examined by enzyme immunoassay and PCR, 160 patients with positive results for HCV with previously determined genotypes were chosen. For the control group, 160 first time blood donors referred to the Regional Blood Transfusion organization of the West Azerbaijan province, northwestern Iran were selected. Oxidative stress markers such as total antioxidant status (TAS), serum levels of reduced (GSH) and oxidized (GSSG) glutathione, Gamma-glutamyl transferase (GGT) and malondialdehyde (MDA) were evaluated in patients infected with diverse HCV genotypes and those in the control group.

Results: In the patient and control groups, the mean \pm SE of TAS, GSH, GSSG, GGT and MDA were $1.04 \pm 0.35$ vs. $2.68 \pm 0.77,1.25 \pm 0.37$ vs. 3.12 $\pm 0.58,0.20 \pm 0.05$ vs. $0.08 \pm 0.04,26.82 \pm 5.62$ vs $8.28 \pm 2.03$ and $2.56 \pm 0.60$ vs. $0.93 \pm 0.34$. All markers had statistical difference between the two groups $(\mathrm{P}<0.05)$. Obvious differences were found in oxidant/antioxidant balance among diverse HCV genotypes with an ascending trend in antioxidant levels among patients infected with genotypes 1a/b, 4, 2a/c, 2b, 3a and healthy controls and a vice versa trend in measures of oxidative markers except for malondialdehyde with a variable pattern.

Conclusions: More serious disease in HCV genetic subtype 1a/1b might be associated with more severe oxidative stress. Milder damage in subtypes 4, 2a/c, 2b and 3a could be related to lower oxidative response, respectively. Acombination of antiviral and antioxidative therapies may enhance the overall response rate of patients with HCV infection, especially with more destructive genotypes.
\end{abstract}

Keywords: Antioxidant; Glutathione; Gamma-Glutamyl Transferase; Malondialdehyde; Hepatitis C

\section{Background}

Infection with hepatitis C virus [(Family Flaviviridae, Genus Hepacivirus, Species Hepatitis C virus), (HCV)] has become a global health problem. The WHO estimate of the prevalence of $\mathrm{HCV}$ infection is $2 \%$, representing 123 million people worldwide (1). This single-stranded RNA virus causes both acute and chronic hepatitis. Although Chronic HCV is a benign viral infection in most patients, it has been known as the principal cause of cirrhosis, hepatocellular carcinoma, extrahepatic diseases and the leading indication for liver transplantation in the developed world (2-4).

The molecular mechanism of HCV pathogenesis and the cause of progression of liver disease to severe liver injury are still poorly understood. Oxidative stress has emerged as a key player in the development and pathogenesis of chronic $\operatorname{HCV}(2,5,6)$. HCV displays a remarkable degree of genomic diversity and background genotyping of HCV has become an essential tool for prognosis and prediction of treatment duration (7). At least 11 genotypes and 70 subtypes were described worldwide from which five
HCV genotypes (1a/b, 2a/c, 4, 2b and 3a) were reported in West Azerbaijan province $(8,9)$. It has been reported that sensitivity to antiviral therapy varies between genotypes and even within each genotype (10-12).

\section{Objectives}

To the best of authors' knowledge, there are few investigations regarding oxidative status and background genotyping. Thus, in the current study, total antioxidant status (TAS), serum levels of reduced (GSH) and oxidized (GSSG) glutathione, Gamma-glutamyl transferase (GGT) and malondialdehyde (MDA) were analyzed in patients infected with diverse HCV genotypes.

\section{Patients and Methods}

\subsection{Patients}

A case-control study was performed on outpatients referred to a private medical laboratory in the West Azerbaijan province, northwestern Iran. From 2006 to 2008,

Copyright ( C) 2015, Kowsar Corp. This is an open-access article distributed under the terms of the Creative Commons Attribution-NonCommercial 4.0 International License (http://creativecommons.org/licenses/by-nc/4.0/) which permits copy and redistribute the material just in noncommercial usages, provided the original work is properly cited. 
of 310 examined patients whose blood samples were tested with both enzyme immunoassay (EIA) and PCR, 128 (41.3\%) had negative results for EIA.Among 182 remaining patients with positive resultsfor EIA, 22 had negative results in PCR. Therefore, 160 patients with positive results by both PCR and EIA method with determined genotypes (9) were selected. The inclusion criteria were adult patients [age 20-50 years, either gender (males or females)] who were new cases and had normal liver transaminases. The exclusion criteria were patients with other chronic medical disorders or with previous HCV treatment history. Besides, those who refused to participate were excluded from the study. The control group consisted of 160 age- and gender-matched blood donors referred to the Regional Blood Transfusion Organization. Ethical approval to perform this study was obtained from the local ethics committee of Urmia pathobiology center. Serum of patients and control group was separated using bench type centrifugation method and frozen at $-40{ }^{\circ} \mathrm{C}$ for future oxidative status evaluation by one observer.

\subsection{Total Antioxidant Status}

Serum TAS was measured usinga Hitachi Analyzer with a Randox reagent kit (Randox Laboratories Ltd, UK). Control samples were obtained from the same company. The determination is based on the reaction of ABTS (2,2-Azinodi-[3-ethylbenzthiazoline sulfonate]) with a peroxidase (metmyoglobin) and $\mathrm{H}_{2} \mathrm{O}_{2}$ to produce the radical cation ABTS+. Antioxidants in the serum suppress cation ABTS+ to a degree that is proportional to their concentration. This cation has a fairly stable blue-green color, which is measured at $600 \mathrm{~nm}$.

\subsection{Reduced and Oxidized Glutathione}

GSH concentration was determined using a Microplate Assay kit (Oxford Biomedical Research Inc.) and an Awerness microplate reader (Molecular Devices, Sunnyvale, CA). In this assay, the reaction of GSH with Ellman's reagent (5,5'-dithiobis-2-nitrobenzoic acid (DTNB)) gives rise to a product that can be quantified spectrophotometrically at $412 \mathrm{~nm}$. This reaction is used to measure the reduction of GSSG to GSH. The rate of reaction is proportional to GSH and GSSG concentrations.

\subsection{Gamma-Glutamyl Transferase}

Serum GGT was measured by commercial kit (Pars Azmoon, Tehran, Iran) using an autoanalyzer (Biotecnica, Targa 3000, Rome, Italy). Control serum (Randox control sera, Antrim, UK) was used to control the measurement accuracy. The GGT catalyses the transfer of $\gamma$-glutamyl moiety of L- $\gamma$-glutamyl-3-carboxy-4-nitranilide to glycylglycine, whereby L- $\gamma$-glutamyl-glycylglycine + 5-amino2-nitrobenzoate are formed. Formation of 5-amino-2-nitrobenzoate, which serves as a measure of GGT activity was measured by spectrophotometry at $405 \mathrm{~nm}$.

\subsection{Malondialdehyde}

MDA was measured by the modified thiobarbituric acid method described by Buege and Aust (13). In this method, thiobarbituric acid reactive substances concentration was calculated using $1.56 \times 10^{-5} \mathrm{M}^{-1} \mathrm{~cm}^{-1}$ as mol/L extraction coefficient.

\subsection{Statistical Analysis}

SPSS 16 (SPSS for windows ver.16) was used for statistical evaluation. One sample Kolmogorov-Smirnov test was used to assess the distribution characteristics of variables. Quantitative data were assessed using ANOVA with Tukey's post hoc comparison of the means. Results were reported as mean \pm SD. Probability values of less than 0.05 were considered as statistically significant.

\section{Results}

In the patient and control groups, the mean \pm SE of TAS, GSH, GSSG, GGT and MDA were $1.04 \pm 0.35$ vs. $2.68 \pm 0.77,1.25$ \pm 0.37 vs. $3.12 \pm 0.58,0.20 \pm 0.05$ vs. $0.08 \pm 0.04,26.82 \pm 5.62$ vs. $8.28 \pm 2.03$ and $2.56 \pm 0.60$ vs. $0.93 \pm 0.34(\mathrm{P}<0.05$ for all markers). Previous genotyping results are displayed in Table 1(9). The values of oxidative stress parameters in divers HCV genotypes are summarized in Table 2. As shown in this table, serum oxidative stress indicators were markedly enhanced and measures of antioxidants significantly declined in patients infected with HCV compared to control. Besides, measures of oxidative stress markers had similar patterns of variation in patients infected with diverse HCV genotypes. A decreasing trend was observed in the levels of TAS and GSH between patients infected with genotypes 1a/b, 4, 2a/c, 2b and 3a. In contrast, genotype 1a/b had highest levels of serum GSSG, GGT and MDA.

\subsection{Total Antioxidant Status}

Data analysis by one-way ANOVA revealed a significant difference in TAS serum level between the groups ( $\mathrm{F}=$ 141.09, $\mathrm{P}=0.00)$. HCV-3a infected patients had the highest level of serum TAS inpatients groups (Table 2 and Figure 1).

\subsection{Reduced Glutathione}

The biochemical level of GSH in diverse HCV genotypes as well as the control group were significantly different $(\mathrm{F}=$ $246.84, \mathrm{P}=0.00$ ) with group $3 \mathrm{a}$ and $1 \mathrm{a} / \mathrm{b}$ having the highest and lowest values, respectively (Table 2 and Figure 2).

\begin{tabular}{lc}
\hline Table 1. Genotyping Frequencies of HCV Positive Patients \\
\hline HCV Genotype & Frequency $(\%)$ \\
\hline 3a & $77(24.8)$ \\
$\mathbf{2 b}$ & $35(11.3)$ \\
$\mathbf{2 a} / \mathbf{c}$ & $19(6.1)$ \\
$\mathbf{4}$ & $16(5.2)$ \\
$\mathbf{1 a} / \mathbf{b}$ & $13(4.2)$ \\
\hline
\end{tabular}


Table 2. Comparison of Indices of Oxidant-Antioxidant Status inPatients Infected With Diverse HCV Genotypes and Healthy Controls a,b,c

\begin{tabular}{lccccccc}
\hline HCV genotypes & Control & $\mathbf{3 a}$ & $\mathbf{2 b}$ & $\mathbf{2 a} / \mathbf{c}$ & $\mathbf{4}$ & $\mathbf{1 a} / \mathbf{b}$ & P Value \\
\hline TAS, mmol/L & $2.68 \pm 0.77$ & $1.75 \pm 0.64$ & $0.63 \pm 0.12$ & $0.31 \pm 0.08$ & $0.21 \pm 0.13$ & $0.07 \pm 0.02$ & 0.000 \\
GSH, $\mu \mathrm{mol} / \mathbf{L}$ & $3.12 \pm 0.58$ & $1.74 \pm 0.48$ & $1.06 \pm 0.43$ & $0.87 \pm 0.13$ & $0.75 \pm 0.32$ & $0.05 \pm 0.02$ & 0.000 \\
GSSG, $\mu \mathrm{mol} / \mathbf{L}$ & $0.08 \pm 0.04$ & $0.19 \pm 0.08$ & $0.18 \pm 0.03$ & $0.23 \pm 0.03$ & $0.25 \pm 0.04$ & $0.31 \pm 0.03$ & 0.000 \\
GGT, U/L & $8.28 \pm 2.03$ & $19.19 \pm 4.16$ & $26.70 \pm 5.87$ & $27.61 \pm 6.81$ & $36.15 \pm 7.73$ & $59.79 \pm 9.37$ & 0.000 \\
MDA, $\mathbf{~ m o l} / \mathbf{L}$ & $0.93 \pm 0.34$ & $2.44 \pm 0.62$ & $2.37 \pm 0.59$ & $2.79 \pm 0.62$ & $2.48 \pm 0.54$ & $3.62 \pm 0.67$ & 0.000 \\
\hline
\end{tabular}

a Abbreviations: GGT,gamma-glutamyl transferase; GSH, reduced glutathione; GSSG, oxidized glutathione; MDA, malondialdehyde;TAS, total antioxidant status.

$\mathrm{b}$ Data are expressed as mean $\pm \mathrm{SD}$.

${ }^{\mathrm{C}}$ Compared using ANOVA test.

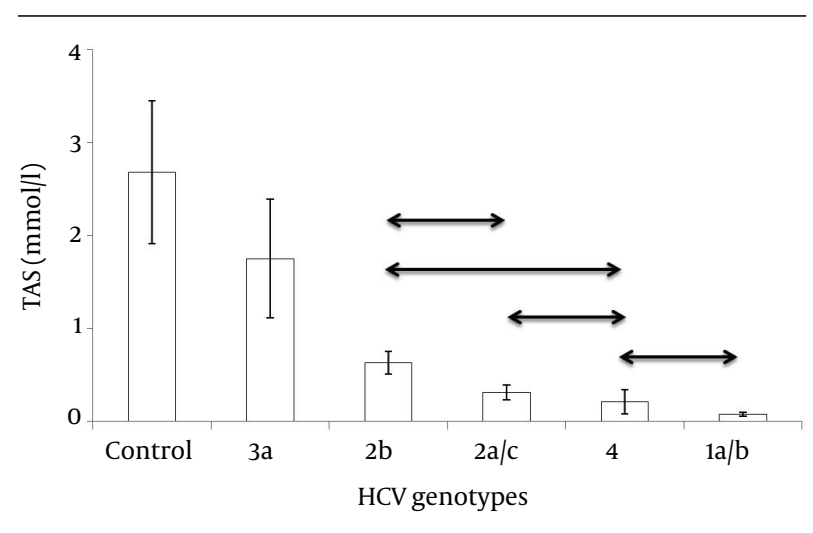

Figure 1. Total antioxidant status (TAS) in serum ofpatients infected with diverse HCV genotypes and the control group. Data are expressed as mean \pm SD. Double arrows indicate groups of means that do not differ based on Tukey's post hoc tests for significant ANOVA results.

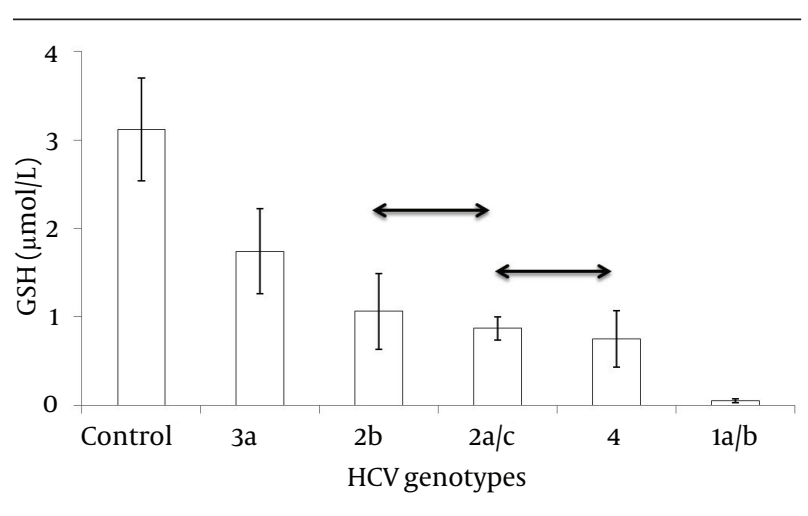

Figure 2. Reduced glutathione (GSH) in serum ofpatients infected with diverse HCV genotypes and control group. Data are expressed as mean \pm SD. Double arrows indicate groups of means that do not differ based on Tukey's post hoc tests for significant ANOVA results.

\subsection{Oxidized Glutathione}

Like other oxidative stress indicators, GSSG level was significantly different in diverse HCV genotypes as well as the control group $(\mathrm{F}=29.48, \mathrm{P}=0.00)$ (Table 2 and Figure 3 ).

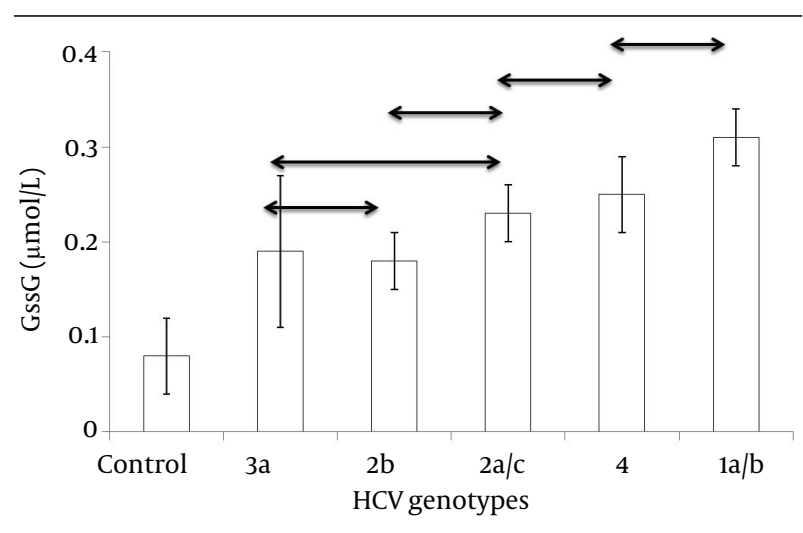

Figure 3. Oxidized glutathione (GSSG) in serum ofpatients infected with diverse HCV genotypes and the control group. Data are expressed as mean \pm SD. Double arrows indicate groups of means that do not differ based on Tukey's post hoc tests for significant ANOVA results.

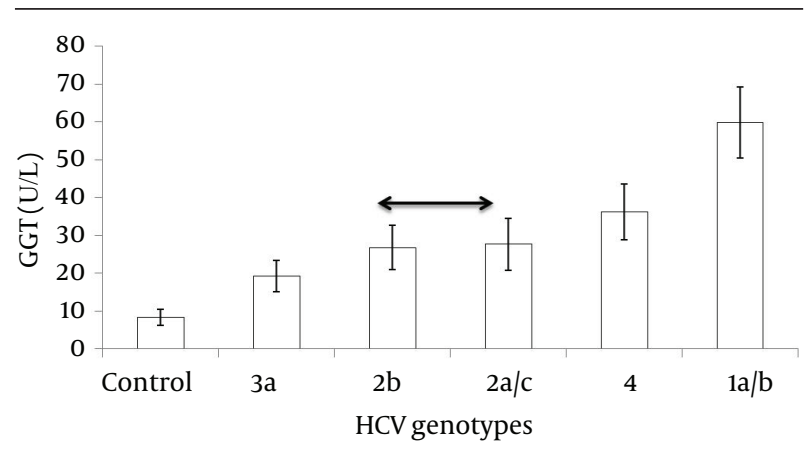

Figure 4. Gamma-glutamyl transferase (GGT) in serum ofpatients infected with diverse HCV genotypes and the control group. Data are expressed as mean \pm SD. Double arrows indicate groups of means that do not differ based on Tukey's post hoc tests for significant ANOVA results.

\subsection{Gamma-Glutamyl Transferase}

There was a significant difference in GGT serum level between the groups $(\mathrm{F}=480.79, \mathrm{p}=0.00)$. A decreasing trend was observed in the levels of GGT inpatients infected with 
genotypes 1a/b, 4, 2a/c, 2b, 3a and healthy controls (Table 2 and Figure 4 ).

\subsection{Malondialdehyde}

Serum MDA concentration was significantly high in all HCV infected patients compared to controls $(F=178.89$, $\mathrm{P}=0.00)$, with group $1 \mathrm{a} / \mathrm{b}$ having a significantly highest level (Table 2 and Figure 5).

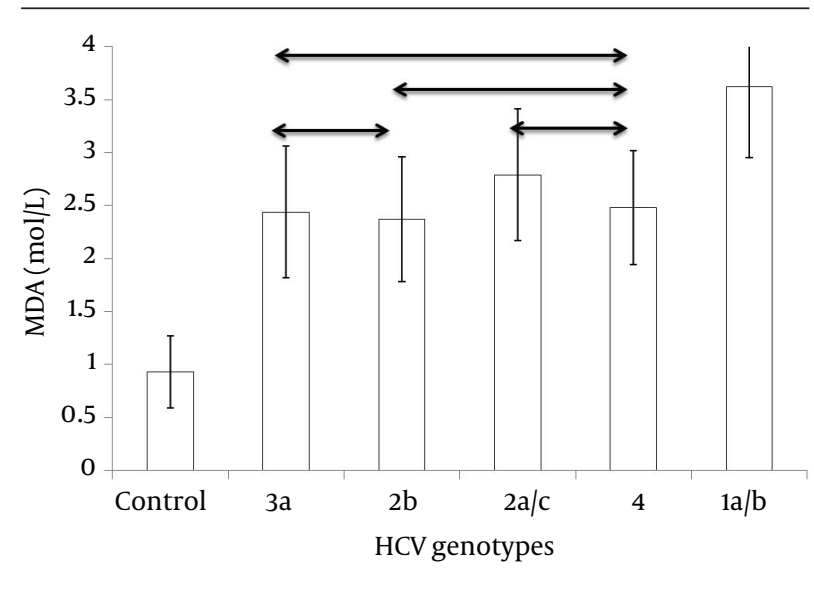

Figure 5. Malondialdehyde (MDA) in serum ofpatients infected with diverse HCV genotypes and the control group. Data are expressed as mean \pm SD. Double arrows indicate groups of means that do not differ based on Tukey's post hoc tests for significant ANOVA results.

\section{Discussion}

Oxidative stress has been proposed as a key step in the development and progression of liver damage $(2,14,15)$. According to the results of this study, there were obvious differences in oxidant/antioxidant balance between diverse HCV genotypes with an ascending trend in antioxidant levels in patients infected with genotypes $1 \mathrm{a} / \mathrm{b}$, $4,2 \mathrm{a} / \mathrm{c}, 2 \mathrm{~b}, 3 \mathrm{a}$ and healthy controls and a vice versa trend in measures of oxidative markers. For unknown reasons, MDA level had a variable pattern with subtle changes among diverse HCV genotypes. Unfortunately, detailed demographic data was unavailable, but the strong point of our study was that PCR-proven HCV infected patients were analyzed and patients were sampled before starting the treatment. Besides, the association of oxidative stress and background genotyping has been described only in few studies. Mahmood et al. found a significant reduction of hepatic, plasmatic and lymphocytic GSH levels in patients chronically infected by HCV, particularly the $1 \mathrm{~b}$ genotype (16). In contrast, Jahan et al. investigated the effects of HCV core protein on cellular genes in hepatocellular carcinoma cell line and reported high expression of oxidative stress related genes in patients infected with HCV genotype 3a compared to 1a (17). This conflict may arise from involvement of host or other viral factors.

Increased oxidative stress and decreased antioxidant levels in chronic hepatitis $\mathrm{C}$ patients have been report- ed in many studies (18-21). Cunningham-Rundles et al. reported that levels of oxidative markers like MDA have been correlated with the severity of chronic hepatitis (22). Because of increased oxidative stress in patients infected with genotypes 1 and 4 in the present study, these genotypes seem to cause more severe disease. Accordingly, Resti et al. reported that HCV genotype 1 is associated with more severe disease, compared with genotypes 2 and 3 (23). Genotypes 2 and 3 also have been reported to be more resistant to antiviral therapy than genotypes 1 and 4 . Besides, long-term response rate in individuals infected with genotypes 1 and 4 has been shown to be much lower than the rates of genotypes 2 and 3(10, 24-26).

Surprisingly and in contrast to the results of current study, steatosis and severe disease has been reported to be more prevalent in patients infected with HCV genotype 3. However, the mechanism involved in HCV induced steatosis is still not clear (27-29).

From the viewpoint of HCV genetic subtypes, Amoroso et al. showed a greater rate of evolution to chronicity and more severe liver disease after acute exposure to HCV genetic subtype $1 \mathrm{~b}$ infection (30). Similarly, some reports proposed a possible link to hepatocellular carcinoma and genetic subtype $1 \mathrm{~b}$. HCV genetic subtype $1 \mathrm{~b}$ also has been mentioned to be associated with earlier recurrence and more severe hepatitis after liver transplantation compared to the other genotypes $(10,31,32)$.

As a result of more severe oxidative stress in HCV genetic subtype $1 \mathrm{a} / \mathrm{1b}$ in the present study, this subtype seems to cause more serious disease in contrast to other subtypes. Although differences in the pathogenicity of genotypes are not clear, genotype has been proved to be one of the key predictors of response to antiviral therapy. Because of genotype-specific differences in response to the new generation of antiviral agents, HCV genotyping can assist in the management of patients and planning for appropriate strategies, particularly the length of treatment $(10,31)$. Moreover, due to the role of oxidative stress in HCV pathogenesis, antioxidants have been proposed in combination with standard treatment regimens (33). It seems that antioxidant supplementation, especially in patients with resistant genotypes, may result in more favorable outcomes. However, Groenbaek et al. showed that supplementation with vitamin $\mathrm{C}, \mathrm{E}$ and selenium increased the antioxidant status, but had no effects on viral load or oxidative markers (34). The efficacy of antiviral treatments particularly in patients with resistant genotypes could be the subject of future studies.

\section{Acknowledgements}

The authors would like to thank members of Urmia pathobiology center for supporting this study. We also appreciate all patients with chronic hepatitis $\mathrm{C}$ and healthy controls who participated in this study. 


\section{Authors' Contributions}

Mohammad Hassan Khadem Ansari and Mir-Davood Omrani developed the original idea, analyzed and interpreted data. Fatemeh Kheradmand contributed to statistical analysis, abstraction of data and preparation of the manuscript.

\section{Funding/Support}

The financial support by Urmia pathobiology center is gratefully acknowledged.

\section{References}

1. Shepard CW, Finelli L, Alter MJ. Global epidemiology of hepatitis C virus infection. Lancet Infect Dis. 2005;5(9):558-67.

2. Simula MP, De Re V. Hepatitis C virus-induced oxidative stress and mitochondrial dysfunction: a focus on recent advances in proteomics. Proteomics Clin Appl. 2010;4(10-11):782-93.

3. Brown RS. Hepatitis $\mathrm{C}$ and liver transplantation. Nature. 2005;436(7053):973-8.

4. Charlton M. Recurrence of hepatitis $C$ infection: Where are we now? Liver Transpl. 2005(11 Suppl 2):S57-62.

5. Choi J, Ou JH. Mechanisms of liver injury. III. Oxidative stress in the pathogenesis of hepatitis C virus. Am J Physiol Gastrointest Liver Physiol. 2006;290(5):G847-51.

6. Vidali M, Tripodi MF, Ivaldi A, Zampino R, Occhino G, Restivo L, et al. Interplay between oxidative stress and hepatic steatosis in the progression of chronic hepatitis C. J Hepatol. 2008;48(3):399406.

7. Nakatani SM, Santos CA, Riediger IN, Krieger MA, Duarte CA, do Carmo Debur M, et al. Comparative performance evaluation of hepatitis $\mathrm{C}$ virus genotyping based on the 5 ' untranslated region versus partial sequencing of the NS5B region of brazilian patients with chronic hepatitis C. Virol J. 2011;8(1):459.

8. Bullock GC, Bruns DE, Haverstick DM. Hepatitis C genotype determination by melting curve analysis with a single set of fluorescence resonance energy transfer probes. Clin Chem. 2002;48(12):2147-54.

9. Omrani MD, Khadem Ansari MH. Hepatitis C Virus Genotyping by Melting Curve Analysis in West Azerbaijan, Northwest of Iran. Hepatitis Monthly. 2009;9(2):133-6.

10. Bowden DS, Berzsenyi MD. Chronic hepatitis C virus infection: genotyping and its clinical role. Future Microbiol. 2006;1(1):103-12.

11. Strahotin CS, Babich M. Hepatitis C variability, patterns of resistance, and impact on therapy. Adv Virol.;2012:267483.

12. Gryadunov D, Nicot F, Dubois M, Mikhailovich V, Zasedatelev A, Izopet J. Hepatitis $C$ virus genotyping using an oligonucleotide microarray based on the NS5B sequence. J Clin Microbiol. 2010;48(11):3910-7.

13. Buege JA, Aust SD. Microsomal lipid peroxidation. Methods Enzymol.1978;52:302-10.

14. Gonzalez-Gallego J, Garcia-Mediavilla MV, Sanchez-Campos S. Hepatitis $C$ virus, oxidative stress and steatosis: current status and perspectives. Curr Mol Med. 2011;11(5):373-90.

15. Madill J, Arendt B, Aghdassi E, Chow C, Guindi M, Therapondos G, et al. Oxidative stress and nutritional factors in hepatitis $C$ viruspositive liver recipients, controls, and hepatitis $C$ virus-positive nontransplant patients. Transplant Proc. 2010;42(5):1744-9.

16. Mahmood S, Kawanaka M, Kamei A, Izumi A, Nakata K, Niiyama G, et al. Immunohistochemical evaluation of oxidative stress markers in chronic hepatitis C. Antioxid Redox Signal. 2004;6(1):19-24.

17. Jahan S, Khaliq S, Ijaz B, Ahmad W, Hassan S. Role of HCV Core gene of genotype 1a and 3a and host gene Cox-2 in HCV-induced pathogenesis. Virol J. 2011;8:155.

18. Duygu F, Koruk ST, Karsen H, Aksoy N, Taskin A, Hamidanoglu M Prolidase and oxidative stress in chronic hepatitis C. J Clin Lab Anal. 2012;26(4):232-7.

19. Venturini D, Simao AN, Barbosa DS, Lavado EL, Narciso VE, Dichi I, et al. Increased oxidative stress, decreased total antioxidant capacity, and iron overload in untreated patients with chronic hepatitis C. Dig Dis Sci. 2010;55(4):1120-7.

20. Abdalla MY, Ahmad IM, Spitz DR, Schmidt WN, Britigan BE Hepatitis $C$ virus-core and non structural proteins lead to different effects on cellular antioxidant defenses. J Med Virol. 2005;76(4):489-97.

21. Korenaga M, Wang T, Li Y, Showalter LA, Chan T, Sun J, et al. Hepatitis $C$ virus core protein inhibits mitochondrial electron transport and increases reactive oxygen species (ROS) production. $J$ Biol Chem. 2005;280(45):37481-8.

22. Cunningham-Rundles S, Ahrn S, Abuav-Nussbaum R, Dnistrian A. Development of immunocompetence: role of micronutrients and microorganisms. Nutr Rev. 2002;60(5 Pt 2):S68-72.

23. Resti M, Jara P, Hierro L, Azzari C, Giacchino R, Zuin G, et al. Clinical features and progression of perinatally acquired hepatitis $C$ virus infection. J Med Virol. 2003;70(3):373-7.

24. Pawlotsky JM. Mechanisms of antiviral treatment efficacy and failure in chronic hepatitis C. Antiviral Res. 2003;59(1):1-11.

25. Zeuzem S. Heterogeneous virologic response rates to interferon-based therapy in patients with chronic hepatitis C: who responds less well? Ann Intern Med. 2004;140(5):370-81.

26. Strahotin CS, Babich M. Hepatitis C variability, patterns of resistance, and impact on therapy. Adv Virol. 2012:267483.

27. Koike K, Moriya K. Metabolic aspects of hepatitis C viral infection: steatohepatitis resembling but distinct from NASH.J Gastroenterol. 2005;40(4):329-36.

28. Asselah T, Rubbia-Brandt L, Marcellin P, Negro F. Steatosis in chronic hepatitis C: why does it really matter? Gut. 2006;55(1):123-30.

29. Idrees M, Riazuddin S. Frequency distribution of hepatitis C virus genotypes in different geographical regions of Pakistan and their possible routes of transmission. BMC Infect Dis. 2008;8:69.

30. Amoroso P, Rapicetta M, Tosti ME, Mele A, Spada E, Buonocore S, et al. Correlation between virus genotype and chronicity rate in acute hepatitis C. J Hepatol.1998;28(6):939-44.

31. Zein NN. Clinical significance of hepatitis $\mathrm{C}$ virus genotypes. Clin Microbiol Rev. 2000;13(2):223-35.

32. Hnatyszyn HJ. Chronic hepatitis $C$ and genotyping: the clinical significance of determining HCV genotypes. Antivir Ther. 2005;10(1):1-11.

33. Melhem A, Stern M, Shibolet O, Israeli E, Ackerman Z, Pappo O, et al. Treatment of chronic hepatitis $C$ virus infection via antioxidants: results of a phase I clinical trial. J Clin Gastroenterol. 2005;39(8):737-42.

34. Groenbaek K, Friis H, Hansen M, Ring-Larsen H, Krarup HB. The effect of antioxidant supplementation on hepatitis $C$ viral load, transaminases and oxidative status: a randomized trial among chronic hepatitis C virus-infected patients. Eur J Gastroenterol Hepatol. 2006;18(9):985-9. 